\title{
Estrategia para la Apropiación de Conocimiento Aplicado a la Formación por Competencias en la Educación Superior
}

\author{
Laura Gallego ${ }^{1}$ y Oscar Araque ${ }^{2}$ \\ (1) Facultad de Contaduría Pública, Universidad Cooperativa de Colombia, sede Ibagué-Espinal 730001, \\ Colombia (e-mail: laura.gallego@campusucc.edu.co) \\ (2) Departamento de Ingeniería Mecánica, Universidad de Ibagué, Ibagué 730001, Colombia \\ (e-mail: oscar.araque@unibague.edu.co)
}

Recibido Jun. 15, 2018; Aceptado Ago. 23, 2018; Versión final Oct. 4, 2018, Publicado Abr. 2019

\begin{abstract}
Resumen
Se establecen los elementos metodológicos que favorecen el desarrollo de competencias en el aula. Para la validación de los elementos metodológicos propuestos se toma como muestra objeto de estudio dos instituciones de educación superior. En la primera institución $(A)$ se toma como referente un curso de primer semestre del área de ciencias económicas, y en la segunda institución (B) se toma como referente un curso de sexto semestre de la facultad de Ingeniería. Utilizando el modelo estadístico de distribución de Gauss se determina la densificación concentrada en los índices superiores de valoración para los periodos en los cuales se aplican los elementos propuestos. Se concluye que los elementos que mayor influencia tienen en la aplicación del modelo por competencias son la integración del proyecto educativo institucional en la metodología y la participación de los actores del conocimiento por medio del trabajo colaborativo.
\end{abstract}

Palabras clave: elementos metodológicos; competencias; herramientas pedagógicas

\section{Strategy for the Appropriation of Knowledge Applied to Competences Model in Higher Education.}

\begin{abstract}
The methodological elements that allow the development of competences in the classroom are established. For the validation of the proposed methodological elements, two institutions of higher education are taken as study cases. In the first institution (A) a first-semester course in the area of Economic Sciences is taken as a reference, and in the second institution (B) a sixth semester course of the Engineering faculty is taken as a reference. The densification concentrated in the higher valuation indexes for the periods where the proposed elements are applied is determined using the statistical model of Gaussian distribution. It is concluded that the elements that have greater influence in the application of the model by competences are the integration of the institutional educational project in the methodology and the participation of the knowledge actors through collaborative work.
\end{abstract}

Keywords: methodological elements; competencies; pedagogical tools 


\section{INTRODUCCIÓN}

Los procesos de investigación y aprendizaje son la base del desarrollo de conocimiento y la etapa primaria de este proceso es el desarrollo del mismo en el aula de ahí, que el constante perfeccionamiento de las técnicas académicas exige la transformación del que hacer docente especialmente en la educación superior, los constantes cambios en los procesos de enseñanza aprendizaje y el acceso cada vez mayor de los estudiantes a la educación superior (Zambrano et al. 2018), han favorecido el desarrollo de metodologías orientadas a la construcción de conocimiento, en este contexto es necesario identificar las capacidades que estos aportan para la construcción de nuevos saberes, de lo anterior, diversos investigadores se han cuestionado si es necesario replantear los modelos tradicionales de enseñanza y aprendizaje por estrategias que involucren transformaciones en las diversas tareas y promueven la calidad en la enseñanza (Lambrechts et al. 2013), al respecto Antón y Pérez (2015), plantea que los modelos tradicionales de enseñanza se basan principalmente en la formación a partir de clases magistrales, la exposición de conceptos y el desarrollo de ejercicios por parte de los estudiantes, lo cual requiere de un nivel de atención superior en el primer instante y posteriormente repetición mas no apropiación del nuevo conocimiento, lo cual se traduce en que estos saberes son rápidamente olvidados y por ende no son aprovechados como debe ser el verdadero objetivo de las escuelas de enseñanza en todos los niveles.

De aquí resulta la siguiente pregunta ¿es posible encontrar un equilibrio entre los diversos métodos de enseñanza aprendizaje que garanticen la apropiación real de conocimiento? Este cuestionamiento es especialmente relevante debido a que las instituciones de formación superior involucran diversos modelos de educación que han sido exitosos, sin embargo en la actualidad los modelos de enseñanza basados en la formación por competencias han cobrado mayor relevancia, estos fueron propuestos hace varios años pero han sido incorporados a los pensum académicos solo recientemente (Marsh, 1987), este modelo de formación se fundamenta en dos corrientes teóricas, el cognitivismo y el constructivismo, el primero se ocupa de la forma en que el estudiante adquiere y aplica el conocimiento y las habilidades, promueve la construcción del conocimiento a partir de la simbiosis de los elementos cognitivos y meta cognitivos del proceso de aprendizaje por medio de la reflexión que lleva al desarrollo del conocimiento (Tardif, 1992). Mientras tanto el modelo constructivista que apoya la formación por competencias se fundamenta en el papel del estudiante como artífice de su conocimiento, sostiene que la apropiación de nuevo conocimiento está relacionada con los conocimientos previos y fomenta los procesos de autoaprendizaje a partir del ser, saber y saber hacer (Ponsa et al. 2009). Sin embargo la implementación de este modelo de formación en el aula ha dejado algunas experiencias negativas, en la Tabla 1 se muestran algunas tipologías que al respecto según los investigadores Palominos et al. (2014), atribuyen a la inefectividad del modelo de competencias.

Tabla 1: Causas de la ineficacia del modelo de competencias.

\begin{tabular}{|l|l|}
\hline \multicolumn{1}{|c|}{ Causa / pre- conceptos errados } & \multicolumn{1}{c|}{ Consecuencia } \\
\hline $\begin{array}{l}\text { El modelo metodológico de competencias } \\
\text { resuelve las debilidades curriculares }\end{array}$ & $\begin{array}{l}\text { Este es un paradigma no acertado, los currículos deben } \\
\text { ser integrales y fortalecidos a partir de la interacción de los } \\
\text { actores del proceso de enseñanza y aprendizaje }\end{array}$ \\
\hline $\begin{array}{l}\text { La reformulación de los programas } \\
\text { académicos se realiza sin el asesoramiento } \\
\text { adecuado }\end{array}$ & $\begin{array}{l}\text { Se presentan currículos poco integradores, que no } \\
\text { responden a las necesidades actuales de la formación } \\
\text { profesional o sesgados a saberes caducos. }\end{array}$ \\
\hline $\begin{array}{l}\text { La reformulación de los programas } \\
\text { académicos no posee el apoyo institucional }\end{array}$ & $\begin{array}{l}\text { Poca efectividad de las acciones soportadas, } \\
\text { exclusivamente en el interés de un grupo de entusiastas } \\
\text { académicos. }\end{array}$ \\
\hline $\begin{array}{l}\text { No se considera la integralidad del proceso } \\
\text { de formación por competencias dejando de } \\
\text { lado la evaluación. }\end{array}$ & $\begin{array}{l}\text { Esto provoca que el momento cumbre en la evaluación de } \\
\text { los saberes no cuente con instrumentos adecuados para el } \\
\text { nivel de formación o la técnica implementada no sea la } \\
\text { adecuada. }\end{array}$ \\
\hline $\begin{array}{l}\text { La sola inclusión de tecnologías de la } \\
\text { información y comunicación (TICs) aporta } \\
\text { soluciones al problema de la apropiación del } \\
\text { conocimiento y la aplicación de } \\
\text { competencias. }\end{array}$ & $\begin{array}{l}\text { Es necesario construir puentes entre la formación } \\
\text { tradicional y la implementación de TICs, lo contrario puede } \\
\text { derivar en desatención del estudiante o mala orientación } \\
\text { de los recursos tecnológicos. }\end{array}$ \\
\hline
\end{tabular}

Por lo anterior es necesario plantear estrategias que involucren a los diversos actores presentes en los modelos de enseñanza tradicional, articulando los elementos presentes en el currículo, con el desarrollo de actividades integradoras en el aula y la evaluación de los resultados que valoren de forma efectiva el aprendizaje logrado. El objetivo de este artículo está orientado a la identificación de los elementos 
metodológicos que mayor influencia tienen en la apropiación de conocimiento basado en modelos pedagógicos ampliamente aceptados y verificar su implementación en cursos de formación superior de las áreas de ciencias económicas e ingeniería en dos instituciones universitarias de Colombia, de esta forma valorar la implementación de las metodologías previstas y compararlas con los resultados históricos obtenidos en las asignaturas objeto de estudio. El aporte científico consiste en describir técnicas aplicadas a cursos de formación profesional, desarrollados a partir de actividades implementadas en el aula e identificar estrategias validadas con el propósito de ser discutidas en busca de su perfeccionamiento.

A partir de la implementación del enfoque por competencias en los cursos de formación profesional los docentes se han visto obligados a apropiar herramientas que anteriormente eran de difícil acceso o por completo desconocidas, pero que han empezado a incursionar fuertemente gracias a los avances tecnológicos y el acceso a recursos educativos abiertos (REA), que de acuerdo a la definición propuesta por la UNESCO (2002) son materiales digitalizados ofrecidos de forma libre y abierta a educadores, estudiantes y autodidactas para utilizar y re-utilizar en la enseñanza, el aprendizaje y la educación o investigación; entre la gran cantidad de material se encuentran los libros electrónicos, las paginas interactivas y comerciales, laboratorios virtuales y las aplicaciones de apoyo académico. El investigador Yorke (2003), ha establecido que en gran medida la utilización de estas metodologías se ven limitadas por el nivel de preparación de los docentes al momento de realizar la aplicación en el aula y la brecha generacional existente. Sin embargo Trillo (2012) y Coates et al. (2005), han comunicado experiencias exitosas de implementación de este tipo de técnicas, concluyen que la extensión de las actividades es un factor determinante para el éxito de la estrategia, así mismo el establecer recursos con patrones de uso cíclico apoya el acondicionamiento de la técnica y la apropiación de la herramienta.

Otra alternativa pedagógica utilizada para favorecer los procesos de enseñanza y aprendizaje es el desarrollo de prototipos, entendiéndose este como un modelo construido con el propósito de representar conceptos funcionales y construir ideas o conceptos a partir de la experimentación practica y el mejoramiento o desarrollo del mismo, integrando nuevas funciones o adaptaciones que lo hagan mejor; al respecto los investigadores Gutiérrez et al. (2016), indican que el desarrollo de este tipo de artefactos facilita el aprendizaje y conceptualización de aspectos técnicos de la formación en ciencias aplicadas. De igual forma facilita el desarrollo de nuevo conocimiento a partir de la experimentación y adaptabilidad que se logra en la construcción de los mismos. Con el propósito de integrar estas propuestas metodológicas en el presente artículo se identifican los elementos metodológicos que permiten el avance en la apropiación de conocimientos incorporando los antes descritos e involucrando otros como el aprendizaje colaborativo que según Wheeler et al. (2008), es una propuesta de enseñanza-aprendizaje basada en los conceptos de cooperación, trabajo grupal, comunicación y responsabilidad. El desarrollo de las actividades debe contar con la colaboración de todos los integrantes del grupo verificando su ejecución y evaluando la calidad y veracidad de las tareas elaboradas. La principal diferencia que existe con el aprendizaje grupal, es que en el aprendizaje colaborativo no existe un líder, todos los miembros del grupo actúan como líderes de las tareas que le han sido designadas y el éxito de la tarea depende del éxito individual de cada uno de los miembros del equipo.

\section{METODOLOGIA}

El enfoque educativo por competencias requiere que su implementación esté ligada al modelo pedagógico integrado al proyecto educativo institucional (PEI) según el investigador Pirela et al. (2014), el enfoque de las competencias contribuye a ampliar la pertinencia de los programas académicos orientando el aprendizaje con el contexto social, disciplinar y cultural en el que se encuentra inmerso el programa de formación profesional. Para llevar a cabo esta investigación y establecer la influencia de los elementos metodológicos objeto de estudio se utilizó un enfoque de investigación cuantitativo (Hussein, 2015) mediante el desarrollo de actividades de valoración de los elementos utilizados.

\section{Muestra}

Para la selección de la muestra se consideró la técnica de muestreo no probabilístico, debido a que los participantes fueron seleccionados de forma intencionada. Al respecto se ha optado por desarrollar el estudio de la implementación de herramientas pedagógicas en dos instituciones de educación superior que involucran áreas del saber diferentes, en la primera institución (A) se tomó como referente un curso de primer semestre del área de ciencias económicas, en la segunda institución (B) se toma como referente un curso de sexto semestre de la facultad de ingeniería. Los grupos estuvieron compuestos en promedio por 30 individuos, donde para la institución (A) 65\% fueron de género femenino y la edad promedio general fluctúa entre 16 y 18 años, para la institución (B) 73\% fueron de género masculino y la edad promedio general fluctúa entre 18 y 21 años. 


\section{Procedimiento}

En la primera etapa de la investigación se toman los promedios académicos en las asignaturas objeto de estudio para una muestra de cinco (5) años, donde el último año corresponde a la implementación de los elementos metodológicos descritos en la Tabla 2.

Tabla 2: Proceso de implementación de los elementos metodológicos.

\begin{tabular}{|c|c|c|}
\hline $\begin{array}{l}\text { Elemento } \\
\text { metodológico }\end{array}$ & Proceso de validación & Actividades de validación \\
\hline $\begin{array}{l}\text { El modelo } \\
\text { metodológico está } \\
\text { integrado al PEI }\end{array}$ & $\begin{array}{l}\text { Se verifica la existencia de un proyecto } \\
\text { educativo institucional integrador que } \\
\text { favorezca la implementación de } \\
\text { herramientas metodológicas acorde al } \\
\text { desarrollo de competencias }\end{array}$ & $\begin{array}{l}\text { Revisión del PEI e identificación de aspectos } \\
\text { relacionados con la implementación de } \\
\text { currículos académicos flexibles }\end{array}$ \\
\hline $\begin{array}{l}\text { Existencia de recursos } \\
\text { suficientes que } \\
\text { faciliten al acceso a } \\
\text { tecnologías de la } \\
\text { información y } \\
\text { comunicación (TICs) }\end{array}$ & $\begin{array}{l}\text { Verificación de espacios suficientes con } \\
\text { conectividad a redes de comunicación. } \\
\text { Existencia de una cultura institucional } \\
\text { que favorezca el uso de recursos } \\
\text { educativos abiertos (REA). Capacidad } \\
\text { de los docentes y de los estudiantes } \\
\text { para la aplicación de TICs } \\
\text { Contexto de aplicación de los recursos } \\
\text { tecnológicos }\end{array}$ & $\begin{array}{l}\text { Establecer el número de espacios apropiados } \\
\text { para el desarrollo de actividades que } \\
\text { involucren el uso de TICs. Verificar la } \\
\text { conectividad y facilidad de acceso a redes } \\
\text { wifi y bases de datos o RAE. Identificar los } \\
\text { saberes necesarios para abordar el uso de } \\
\text { herramientas tecnológicas RAE }\end{array}$ \\
\hline $\begin{array}{l}\text { Desarrollo de } \\
\text { prototipos para la } \\
\text { experimentación }\end{array}$ & $\begin{array}{l}\text { Recursos apropiados para el desarrollo } \\
\text { de prototipos para el aprendizaje en el } \\
\text { aula } \\
\text { Experiencia de los docentes en } \\
\text { procesos de construcción y desarrollo } \\
\text { de equipos }\end{array}$ & $\begin{array}{l}\text { Existencia de recursos (económicos, } \\
\text { infraestructura y equipos) que favorezca el } \\
\text { desarrollo de prototipos } \\
\text { Determinar los saberes necesarios y } \\
\text { herramientas conceptuales requeridas por } \\
\text { docentes y estudiantes. }\end{array}$ \\
\hline Trabajo colaborativo & $\begin{array}{l}\text { Identificación de técnicas aplicadas a } \\
\text { grupos. Establecer los lineamientos } \\
\text { para la conformación de los grupos de } \\
\text { trabajo }\end{array}$ & $\begin{array}{l}\text { Establecer las tecnologías más apropiadas } \\
\text { para el desarrollo de actividades grupales. } \\
\text { Asignar los roles y normas a los integrantes } \\
\text { del grupo. Definir los objetivos en común } \\
\text { orientados al desarrollo del trabajo en equipo }\end{array}$ \\
\hline $\begin{array}{l}\text { Creación de } \\
\text { conocimiento }\end{array}$ & $\begin{array}{l}\text { Verificar los previos necesarios sobre la } \\
\text { temática tratada. } \\
\text { Construir el estado del arte de la } \\
\text { temática de interés }\end{array}$ & $\begin{array}{l}\text { Identificar referentes (internos o externos) } \\
\text { que orienten el conocimiento hacia la } \\
\text { construcción de saberes. Organizar la } \\
\text { información en orden cronológico e impacto } \\
\text { sobre la temática objeto de estudio }\end{array}$ \\
\hline Evaluación & $\begin{array}{l}\text { Identificación de mecanismos } \\
\text { adecuados de evaluación } \\
\text { Existencia de escenarios apropiados } \\
\text { para el desarrollo de actividades } \\
\text { evaluativas }\end{array}$ & $\begin{array}{l}\text { Existencia de espacios adecuados para el } \\
\text { desarrollo de procesos de evaluación y } \\
\text { aprendizaje. Evaluar la articulación de los } \\
\text { conceptos previos con la construcción de } \\
\text { unos nuevos o mejor fundamentados. } \\
\text { Evaluar el desempeño de los integrantes del } \\
\text { grupo y el aporte individual a la construcción } \\
\text { de saberes grupales. }\end{array}$ \\
\hline $\begin{array}{l}\text { Socialización de } \\
\text { resultados }\end{array}$ & $\begin{array}{l}\text { Existencia de escenarios apropiados } \\
\text { para la socialización de resultados } \\
\text { Establecer procedimientos y normas de } \\
\text { respeto y entendimiento para el proceso } \\
\text { de divulgación de resultados. }\end{array}$ & $\begin{array}{l}\text { Socialización de los alcances logrados, frente } \\
\text { a los miembros de la comunidad académica. } \\
\text { Presentar las evidencias del desarrollo } \\
\text { logrado, en caso de prototipos el nivel de } \\
\text { desarrollo en función de os recursos } \\
\text { disponibles. } \\
\text { Recoger los comentarios positivos y } \\
\text { negativos con el propósito de establecer } \\
\text { mejoras al proceso de construcción de } \\
\text { conocimiento. }\end{array}$ \\
\hline
\end{tabular}

En la segunda etapa de la investigación se realiza una valoración de los elementos metodológicos implementados con los estudiantes del año, estos fueron efectuados (año 5), los elementos metodológicos 
planteados se implementan en una población seleccionada, donde los sujetos no tuvieron interacción al momento de responder la encuesta, las actividades de validación son ponderadas y se establece la media estadística para las dos instituciones de educación superior objeto de estudio. Para el proceso de calificación se propone utilizar el modelo de escala de Likert para un formato de cinco (5) niveles de respuesta como se muestra en la Tabla 3.

Tabla 3: Escala de Evaluación de las actividades de validación.

\begin{tabular}{|l|c|}
\hline \multicolumn{1}{|c|}{ Nivel de conformidad } & Valoración \\
\hline Totalmente en desacuerdo & 1. \\
\hline En desacuerdo & 2. \\
\hline Ni de acuerdo ni en desacuerdo & 3. \\
\hline De acuerdo & 4. \\
\hline Totalmente de acuerdo & 5. \\
\hline
\end{tabular}

\section{RESULTADOS Y DISCUSIÓN}

La investigación se desarrolla utilizando el método de investigación cualitativa con observación participativa debido a que el grupo de investigadores hizo parte de la implementación de los elementos metodológicos evaluados. Los resultados de la ponderación parcial se acercaron al entero más próximo, mientras que para el promedio general se asume el valor de la media aritmética. Para el proceso de implementación se tomó como referencia un año escolar el cual consta de dos periodos semestres académicos (Sem), los resultados históricos se tienen con posterioridad y se asume un periodo de análisis comprendido por los últimos cinco (5) años, las calificaciones se presentan en función de la nota final obtenida en el curso como el promedio académico de todos los estudiantes del curso, lo cual se entiende como el porcentaje de éxito o fracaso de la implementación de la estrategia. A continuación en la Figura 1, se ilustran los resultados obtenidos.

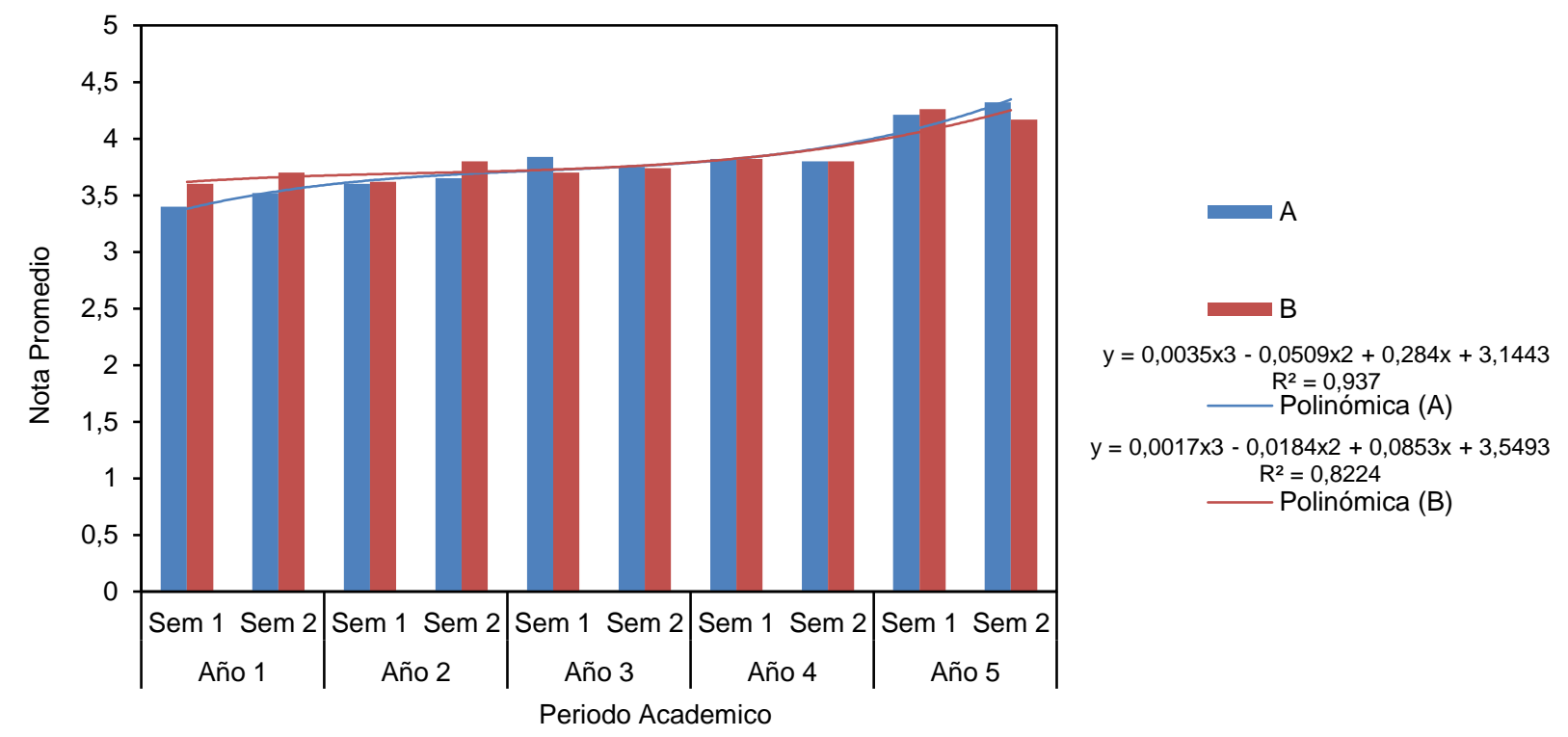

Fig. 1: Notas ponderadas históricas en las dos instituciones

En la Figura 1, se ilustran los resultados históricos medidos como el promedio de las notas obtenidas por los estudiantes de cada uno de los cursos objeto de estudio, se observa que en el periodo académico Año 5, donde se realiza la implementación de los elementos metodológicos propuestos se logra obtener una mejora en los resultados académicos para cada uno de los semestres donde se ejecuta la estrategia, con el propósito de verificar el éxito de la implementación del modelo se obtienen las ecuaciones polinómicas de tendencia con un coeficiente de determinación cercano a uno en los dos casos lo cual indica un modelo ajustado a los escenarios indicados. Para obtener un resultado más objetivo se realiza la comprobación de los resultados individuales para el primer y último periodo académico (Año 5) donde se realiza la implementación de las estrategias y se verifica la función de densidad en una gráfica de distribución normal. Utilizando el modelo propuesto por Carl Friedrich Gauss (1777-1855) e indicado según la ecuación (1) que se muestra a continuación Kanungo et al. (2000). 


$$
f(x)=\frac{1}{\sigma \sqrt{2 \pi}} e^{-\frac{1}{2} \cdot\left(\frac{x-\mu}{\sigma}\right)^{2}}
$$

Donde la característica de $\mathrm{x}$ es que sigue una distribución normal de media $\mu$ y varianza $\sigma^{2}$, obteniéndose una curva en forma de campana asintótica al eje de las abscisas. A continuación en la Figura 2 se muestran los resultados obtenidos para los dos años analizados incluido el año 5, donde se introdujo la aplicación de las herramientas pedagógicas y posterior mejora en el rendimiento de los estudiantes.
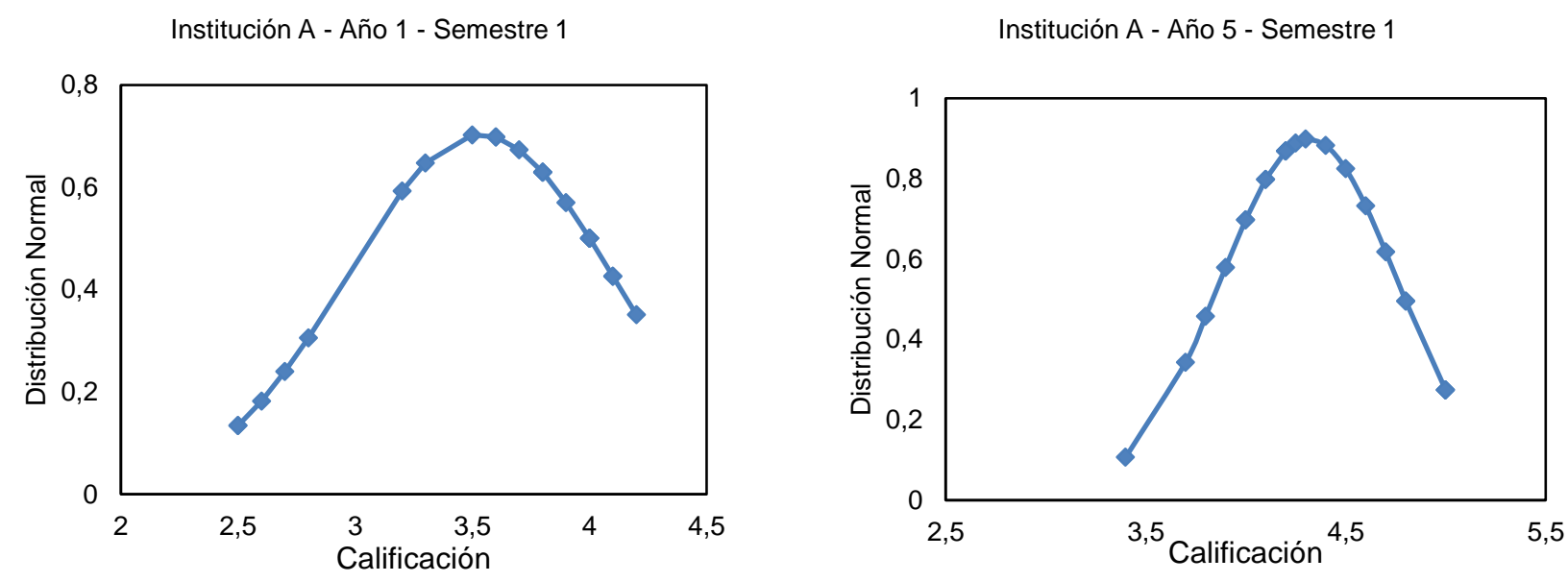

Institución B - Año 1 - Semestre 1

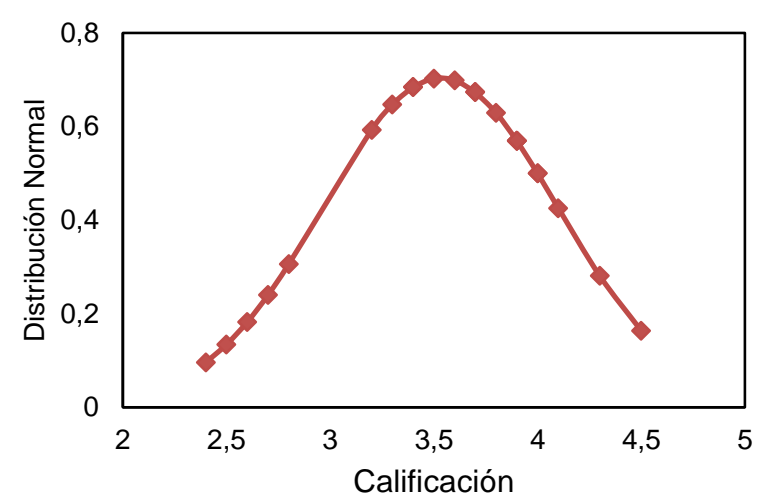

Institución B - Año 5 - Semestre 1

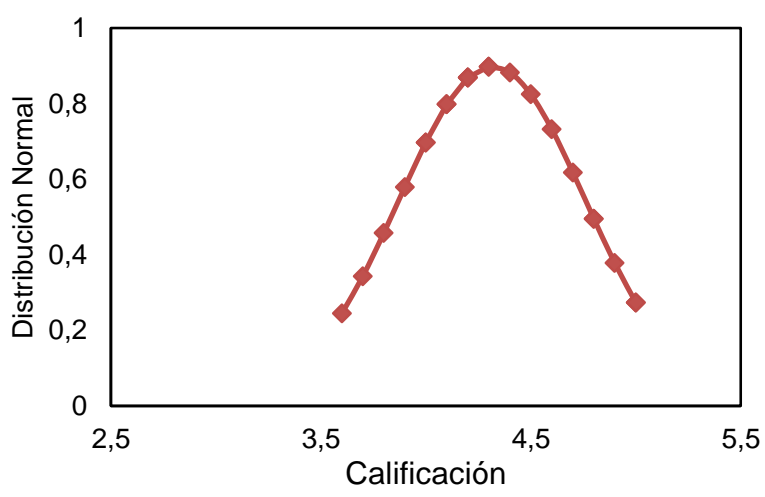

Fig. 2: Notas ponderadas históricas en las dos instituciones

Como se observa en la Figura 2, para cada una de las instituciones de educación superior se obtuvo una mejora en el desempeño académico de los estudiantes se observa que para los primeros años la frecuencia de la calificación estaba alrededor de 3,5 mientras que para los semestres donde se aplicaron las herramientas pedagógicas mencionadas correspondientes al año 5, las notas están agrupadas en los márgenes superiores de la tabla valorativa de calificación, este es un indicativo de mejora académica y apropiación de conocimiento. A partir de los resultados obtenidos para las dos instituciones y la valoración de los elementos metodológicos comunes se muestra la influencia que tienen estos aspectos en el éxito de la aplicación de la propuesta académica.

Los resultados de la ponderación de las actividades de validación para la implementación de los elementos metodológicos se muestran en la Tabla 4. En la tabla 4, $\left(^{*}\right)$ es la Institución (A) Curso de primer semestre de la Facultad de Ciencias Económica; y $\left(^{* *}\right)$ es la Institución (B) Curso de sexto semestre de la Facultad de Ingeniería. Manteniendo el principio de confidencialidad se omite el nombre de las instituciones, los estudiantes y los nombres de las asignaturas que sirvieron de base para el desarrollo de la investigación. De la valoración de los resultados obtenidos para las dos instituciones objeto de estudio y tomando la media estadística de las actividades de validación, en cada institución mostrada en la Tabla 4 se obtienen los datos porcentuales de la Figura 3, indican el nivel de incidencia de cada elemento metodológico en la apropiación de conocimiento, conforme a la percepción de los estudiantes del año cinco (5) donde estas fueron implementadas. 
Tabla 4: Ponderación de las actividades de validación

\begin{tabular}{|l|c|c|}
\hline \multirow{2}{*}{$\begin{array}{c}\text { Elemento metodológico } \\
\text { (Valoración actual) }\end{array}$} & $\begin{array}{c}\text { Valoración de la Media estadística de las actividades } \\
\text { de validación, en cada institución }\end{array}$ \\
\cline { 2 - 3 } & $A^{*}$ & 4 \\
\hline El modelo metodológico está integrado al PEI & 4 & 5 \\
\hline $\begin{array}{l}\text { Existencia de recursos suficientes que faciliten al acceso a } \\
\text { tecnologías de la información y comunicación (TICs) }\end{array}$ & 3 & 4 \\
\hline Desarrollo de prototipos para la experimentación & 2 & 5 \\
\hline Trabajo colaborativo & 5 & 4 \\
\hline Creación de conocimiento & 4 & 4 \\
\hline Evaluación & 5 & 4 \\
\hline Socialización de resultados & 4 & 4,29 \\
\hline Promedio General & 3,86 & 4 \\
\hline
\end{tabular}

En relación a la Figura 3, del modelo por competencias se puede inferir que a mayor trabajo colaborativo, mayor creación de conocimiento, esto se debe a que existe una mayor articulación entre los miembros del equipo de trabajo y de esta forma se incentiva la generación de ideas, las cuales pueden ser favorecidas por la existencia de tecnologías de la información (TICs), como elemento integrado al desarrollo de las actividades, lo cual es coincidente con los planteamientos de Martínez et al. (2018), si la institución tiene un buen nivel de desarrollo (infraestructura y equipos) se logra una injerencia positiva en la obtención y socialización de los resultados, esto se debe a que el uso de TICs favorece el acceso a información que bajo la orientación adecuada será seleccionada favoreciendo la apropiación del conocimiento.

En cuanto al desarrollo de prototipos se evidencio que llegan a tener mayor relevancia en aquellos cursos que poseen un alto componente práctico tecnológico o con niveles técnicos relacionados al desarrollo de saberes, es un elemento deseable pero no imprescindible, que puede ser adoptado por los REA que apoyan el desarrollo en el aula.

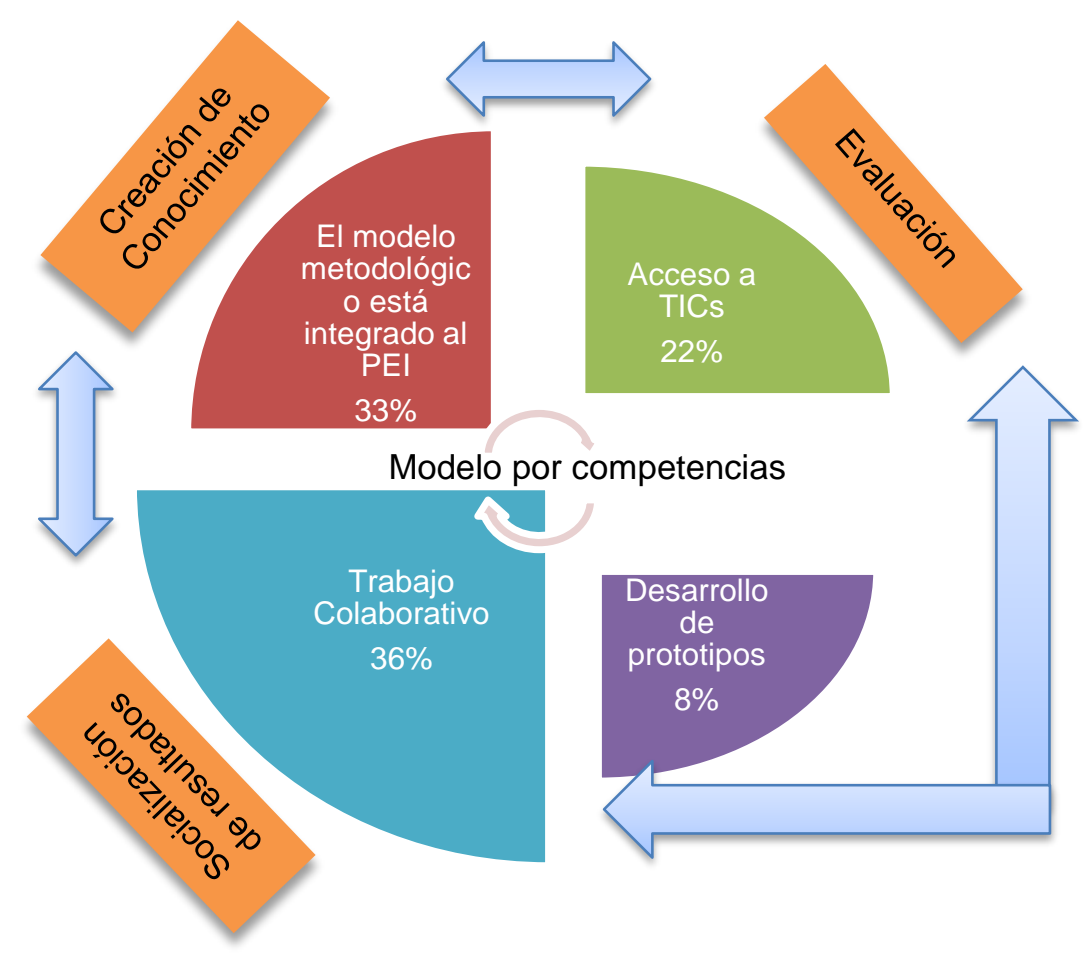

Fig. 3: Influencia de los elementos metodológicos en la aplicación del modelo por competencias

\section{CONCLUSIONES}

En conformidad con los resultados obtenidos se logró establecer los elementos metodológicos que favorecen el desarrollo de competencias en el aula, indicando que aquellos que mayor peso tienen son el trabajo 
colaborativo con una incidencia del $36 \%$ y la existencia del modelo pedagógico por competencias, integrado al proyecto educativo institucional con un $33 \%$ de incidencia. Se evidencio que las instituciones que involucran en el Proyecto Educativo el modelo por competencias, tienen mayor posibilidad de éxito al implementar los elementos metodológicos propuestos, esto se debe a que las condiciones institucionales serán favorables y la adaptabilidad se dará en menor tiempo.

Se plantean herramientas pedagógicas que facilitan la implementación del modelo pedagógico por competencias, como son la utilización de tecnologías de la información y comunicación, el desarrollo de prototipos y el trabajo colaborativo, para la validación de los elementos metodológicos propuestos se tomó como muestra objeto de estudio dos instituciones de educación superior, obteniéndose a partir de un estudio con ponderación histórica a cinco años que a partir de la implementación de los elementos metodológicos propuestos se obtuvieron mejores resultados evidenciados en la calificación promediada.

\section{AGRADECIMIENTOS}

Los autores expresan su agradecimiento a la Dirección de Investigaciones de la U. Cooperativa de Colombia Sede Ibagué - Espinal por el apoyo suministrado para el desarrollo del proyecto de investigación ID-2272 y a la Dirección de Investigaciones de la U. de Ibagué, por el apoyo suministrado para el desarrollo del proyecto de investigación 17-525-SEM.

\section{REFERENCIAS}

Antón García, D. y M. Pérez Suárez, La Docencia Universitaria Novel Como Mejora Metodológica, Opción, 31 (3), 104-124 (2015)

Coates, H., R. James y G. Baldwin, A critical examination of the effects of learning management systems on university teaching and learning, Tertiary Education \& Management, 11(1), 19-36 (2005)

Gutiérrez, D., J. Esneder, E.D. Demoya Correal y O.A. González Torres, Diseño y Construcción de Prototipo para el Aprendizaje del Proceso Técnico de Afilado de Buril, Universidad Pedagógica Nacional, Bogotá, Colombia (2016)

Hussein, A., The Use Of Triangulation In Social Sciences Research: Can Qualitative And Quantitative Methods Be Combined? Journal of comparative social work, 4(1), 1-12 (2015)

Kanungo, T., R.M. Haralick y otros tres autores, A statistical, nonparametric methodology for document degradation model validation, IEEE Transactions on Pattern Analysis and Machine Intelligence, 22(11), 1209-1223 (2000)

Lambrechts, W., I. Mulà y otros tres autores, The integration of competences for sustainable development in higher education: an analysis of bachelor programs in management, J. of Cleaner Production, 48, 65-73 (2013)

Larragueta, S.F. y M. Rodorigo, Repensar la Docencia Universitaria: La Coordinación Como Estrategia Para la Innovación, Opción: Revista de Ciencias Humanas y Sociales, (11), 514-530 (2016)

Marsh, H.W., Students' evaluations of university teaching: Research findings, methodological issues, and directions for future research, International journal of educational research, 11(3), 253-388 (1987)

Martínez-Argüello, L.D., F.J. Hinojo-Lucena e I.A. Díaz, Aplicación de las Tecnologías de la Información y la Comunicación (TIC) en los Procesos de Enseñanza- Aprendizaje por parte de los Profesores de Química, Información Tecnológica, 29(2), 41-52 (2018)

Palominos, F.E., M.A. Mendez y R.A. Barrera, Sistema de Perfeccionamiento Orientado a Competencias para Docentes de la Educación Superior, Formación Universitaria, 7(3), 11-22 (2014)

Pirela Morillo, J., C. Vera y J. de Jesús, El desarrollo de Competencias Informacionales en Estudiantes Universitarios: Experiencia y Perspectivas en dos Universidades Latinoamericanas, Investigación Bibliotecológica, 28(64), 145-172 (2014)

Ponsa, P., B. Amante y otros cuatro autores, Higher education challenges: introduction of active methodologies in engineering curricula, International Journal of Engineering Education, 25(4), 799-813 (2009)

Tardif, J., Pour un Enseignement Stratégique: L'apport de la Psychologie Cognitive [Por una Enseñanza Estratégica: La Aportación de la Psicología Cognitiva], Éditions Logiques, Montreal (1992)

Trillo Miravalle, M., Recursos Educativos en Abierto: evolución y modelos, Foro de Educación, 10(14), 191 -205 (2012)

UNESCO, Forum on the impact of Open Courseware for Higher Education in Developing Countries Final Report (2002)

Wheeler, S., P. Yeomans y D. Wheeler, The good, the bad and the wiki: Evaluating student-generated content for collaborative learning, British journal of educational technology, 39(6), 987-995 (2008)

Yorke, M., Formative assessment in higher education: Moves towards theory and the enhancement of pedagogic practice, Higher education, 45(4), 477-501 (2003)

Zambrano, C., F. Albarran y P. Salcedo, Percepción de Estudiantes de Pedagogía Respecto de la Autorregulación del Aprendizaje, http://dx.doi.org/10.4067/S0718-50062018000300073, Formación Universitaria, 11(3), 73-86 (2018) 